\title{
Characterization of the Embryogenic Tissue of the Norway Spruce Including a Transition Layer between the Tissue and the Culture Medium by Magnetic Resonance Imaging
}

\author{
R. Kořínek ${ }^{1}$, J. Mikulka², J. Hřib ${ }^{3}$, J. Hudec ${ }^{1}$, L. Havel ${ }^{3}$, K. Bartušek ${ }^{1}$ \\ ${ }^{1}$ Institute of Scientific Instruments of the CAS, Kralovopolska, 147, 612 64, Brno, Czech Republic, korinek@isibrno.cz \\ ${ }^{2}$ Brno University of Technology, Faculty of Electrotechnical Engineering and Communication, Department of Theoretical \\ and Experimental Electrical Engineering, Technická, 3082/12, 616 00, Brno, Czech Republic \\ ${ }^{3}$ Mendel University in Brno, Faculty of Agronomy, Department of Plant Biology, Zemedelska, 1, 613 00, Brno, Czech \\ Republic
}

\begin{abstract}
The paper describes the visualization of the cells (ESEs) and mucilage (ECMSN) in an embryogenic tissue via magnetic resonance imaging (MRI) relaxometry measurement combined with the subsequent multi-parametric segmentation. The computed relaxometry maps $T_{1}$ and $T_{2}$ show a thin layer (transition layer) between the culture medium and the embryogenic tissue. The ESEs, mucilage, and transition layer differ in their relaxation times $T_{1}$ and $T_{2}$; thus, these times can be used to characterize the individual parts within the embryogenic tissue. The observed mean values of the relaxation times $T_{1}$ and $T_{2}$ of the ESEs, mucilage, and transition layer are as follows: $1469 \pm 324$ and $53 \pm 10 \mathrm{~ms}, 1784 \pm 124$ and $74 \pm 8 \mathrm{~ms}, 929 \pm 164$ and $32 \pm 4.7 \mathrm{~ms}$, respectively. The multi-parametric segmentation exploiting the $T_{1}$ and $T_{2}$ relaxation times as a classifier shows the distribution of the ESEs and mucilage within the embryogenic tissue. The discussed $T_{1}$ and $T_{2}$ indicators can be utilized to characterize both the growth-related changes in an embryogenic tissue and the effect of biotic/abiotic stresses, thus potentially becoming a distinctive indicator of the state of any examined embryogenic tissue.
\end{abstract}

Keywords: Norway spruce, ESEs, mucilage, transition layer, MRI relaxometry.

\section{INTRODUCTION}

Magnetic resonance imaging (MRI) is a non-invasive method widely applied in the study of molecules. The MRI approach is frequently employed not only in medicine but also in biological, biochemical, and chemical research. Most of the papers describing the use of MRI to investigate plant physiology have emphasized certain aspects of anatomy or functional morphology [1]. In plant biology, MRI supports several major activities, namely, the research of the water and mineral compounds transported within a plant [2], [3], the determination of plant metabolites [4], [5], the investigation of cellular processes [6], and the examination of the growth and development of plants [7]. MRI is also instrumental towards monitoring water changes in early somatic embryos (ESEs). Šupálková et al. [8] examine the influence of the ESEs of spruce treated with cadmium and/or lead ions for twelve days using multi-instrumental analytical procedures; these authors employ image analysis to estimate the growth, a fluorimetric sensor to detect the viability of enzyme-treated ESEs, and the MRI technique to facilitate non-destructive measurement of the volume of the ESEs.
The interaction between the cells plays a fundamental role in the growth and development of multicellular organisms. In this context, let us note that the structural integrity of plants was described by, for example, Šebánek et al. [9]; it may also be mentioned that the extracellular matrix (ECM) issue pertains to Dostál's [10] topic of the structural integrity of plant organism [11].

At the early stage of their development, the ESEs are covered with a special extracellular cell wall layer referred to as the extracellular matrix surface network (ECMSN), [12]. Neděla et al. [13], [14], [15] utilized an environmental scanning electron microscope (ESEM) to describe the ECM and/or ECMSN in conifers (spruce, pine, and fir) at the native stage. Arabinogalactan-proteins (AGPs) are progressively accumulated within the ECMSN, a wellknown marker covering the embryogenic cells during the embryo development. Specific AGPs are essential in somatic embryogenesis and exhibit the capability of directing the development of the cells [16]. It is possible that AGPs may be present as adhesives in the middle lamella to cement the cell-to-cell contact and they may also be involved in the adhesion of the callus cell clumps [17]. Two homogeneous AGPs were purified from the Norway spruce 
callus cells via ion-exchange and gel-permeation chromatography followed by enzymatic treatment [18]. The function of AGPs was recently summarized in a review by Seifert and Roberts [19].

Our article further extends a related, previously published paper [20], where the authors conducted 4 experiments: 1 ) the designing of methods to assess the water amount in relevant somatic embryos; 2) a comparison of the SNR in images acquired at different magnetic flux density values of the basic magnetic field; 3) monitoring the tissue growth via various techniques; and 4) the segmentation of two subjectively distinguishable regions in the tissue, with each of these exhibiting a specific $T_{2}$ relaxation time. Our research report develops the work carried out by Mikulka et al., especially as regards the image processing methodology. In the given context, the aim of the present article is to utilize MRI relaxometry in order to visualize the relaxation times of the early embryogenic tissue (callus) of the Norway spruce.

\section{SUBJECT \& METHODS}

\section{Plant material}

A clone of the somatic embryos (ESEs) of the Norway spruce (Picea abies (L.) Karst.), marked as 2/32, was used in our experiments. The cultures were maintained on a semisolid (Gelrite Gellan Gum, Merck, Germany), half-strength LP medium [21], which underwent further modifications within later research [22]. The concentrations of 2.4dichloro-phenoxyacetic acid (2.4-D) and N6 benzyladenine (BAP) corresponded to 4.4 and $9 \mu \mathrm{M}$, respectively [23]. The $\mathrm{pH}$ value was adjusted to 5.7 - 5.8 before autoclaving $\left(121^{\circ} \mathrm{C}, 100 \mathrm{kPa}, 20 \mathrm{mins}\right.$.). The organic part of the saccharose-free solution was sterilized by filtering through a $0.2 \mu \mathrm{m}$ polyethylensulfone membrane (Whatman, Puradisc 25 AS). We used Petri dishes (50 mm in diameter) for the actual cultivation. The sub-cultivation of the stock cultures was performed in two-week intervals; the stock and experimental cultures were maintained at a temperature of $23 \pm 2{ }^{\circ} \mathrm{C}$ in a cultivation box kept in a dark place.

\section{Isolation of the somatic embryos and mucilage}

Ten days after the last passaging, the embryogenic tissues (calluses) of the Norway spruce were used for the isolation of the somatic embryos from the above-discussed heterogeneous, fully asynchronous culture (suspensor cells and embryogenic cells at different stages of development). The most developed somatic embryos with only a minimum amount of mucilage (ECMSN) on the surface were extracted from the embryogenic culture by means of tweezers and placed into two depressions in an acrylic glass cuboid. The mucilage was obtained from the embryogenic tissue (callus) via automatic tweezers placed in the middle of the embryogenic callus; we acquired the substance through capillary attraction and then pipetted it off. This procedure was repeated several times to provide the amount of mucilage necessary to fill the remaining, empty depressions in the applied acrylic glass cuboid. In this manner, the embryos and mucilage were prepared for the MR relaxometry measurement.

\section{MRI relaxometry}

Magnetic resonance imaging (MRI) is a non-invasive imaging method to supply information about an examined tissue, enabling us to observe the inner part of the tissue without causing any physical damage. The MR image contrast is influenced by several characteristics of the tissues and other materials; such characteristics include the $T_{1}, T_{2}$, and $T_{2}{ }^{*}$ relaxation, spin density, susceptibility, and flow effects. When a magnetized spin system is perturbed from the thermal equilibrium state after radio frequency excitation, then, according to the laws of thermodynamics, the system will return into the state of thermal equilibrium. Practically, in MRI, the measured magnetization vector is equal to the sum of all microscopic magnetic moments in the object. In general terms, the behavior of the magnetization vector over time is defined by the Bloch equation [24]. The longitudinal and transverse relaxation times $T_{1}$ and $T_{2}$ represent the time evolution of the longitudinal and transverse magnetization while the magnetization of water protons reverts back to the equilibrium, following the radio frequency (RF) excitation. The $T_{2}{ }^{*}$ time constant defines the progress of the free induction decay signal. The above-mentioned relaxation times are tissue-specific, and all of them depend on the biophysical and chemical properties of the examined tissue. The image contrast is contingent on the used excitation scheme, frequently referred to as the pulse sequence or technique/method.

The general purpose of the experiment was to perform in situ measurement of the ESEs via MRI relaxometry techniques. To measure the $T_{2}$ relaxation, we applied the fast spin-echo (FSE) technique; the $T_{1}$ relaxation was measured using the inversion recovery fast spin-echo (IRFSE) method. All the described experiments were performed on a $9.4 \mathrm{~T}$ (Bruker) MRI scanner at the Institute of Scientific Instruments, Brno. The MAREVISI (8.2) and MATLAB (R2014b) programs were used for the processing.

\section{Data processing}

A method for the multi-parametric segmentation of MR images of tissue cultures was proposed; this segmentation technique exploits the principle of linear mixture models. The values of the relaxation times in the tissue areas have normal distribution (Gaussian distribution); we therefore chose a Gaussian mixture model (GMM), generally expressible as follows [25]:

$$
p(\boldsymbol{\theta} \mid \mathbf{x})=\sum_{i=1}^{K} \tilde{\phi}_{i} N\left(\tilde{\boldsymbol{\mu}}_{i}, \tilde{\boldsymbol{\Sigma}}_{i}\right)
$$

where the $i$-th component of the vector of random numbers $\mathbf{x}$ with the normal distribution $N$ is characterized by the 
weight $\tilde{\phi}_{i}$, vector of mean values $\tilde{\boldsymbol{\mu}}_{i}$, and covariance matrix $\tilde{\boldsymbol{\Sigma}}_{i}$. The vectors $\tilde{\boldsymbol{\mu}}_{i}$ and $\tilde{\boldsymbol{\Sigma}}_{i}$ are estimated via the maximum likelihood method. Direct calculation is virtually unfeasible, owing to strong non-linearity of the optimized function; for this reason, the estimation of the parameter values was performed using an expectation-maximization (EM) algorithm.

The segmentation of the culture images was executed in two steps. Within the first stage, we used Gaussian mixture models to approximate the data of the isolated ESEs and mucilage; in the next stage, the posterior probabilities of pertinence to one of the two GMM components were assigned to the relaxation values of the images. The final segmented image is obtained after thresholding of the acquired probability images.

\section{Results}

MRI measurements of two embryogenic tissue samples (the Norway spruce) were performed; in this context, we also measured the tissues (the ESEs and mucilage) isolated from the embryogenic tissue. The acquired images were used for the calculation of the $T_{1}$ and $T_{2}$ maps, which provide the contrast between the ESEs, mucilage, and transition layer. The coronal and axial slices were measured with the following parameters: the field of view (FOV) of $20 \times 20 \mathrm{~mm}$; the acquisition matrix size of $256 \times 256$ pixels; the layer thickness of $0.5 \mathrm{~mm}$; and the layer gap of $0.5 \mathrm{~mm}$. The voxel size of $0.078 \times 0.078 \times 0.5 \mathrm{~mm}$ is derived from the above parameters. Fig.1. shows the PD images and $T_{1}, T_{2}$ maps of the coronal images of the embryogenic tissue (samples No. 1). The slices are numbered from the bottom (substrate) to the top.

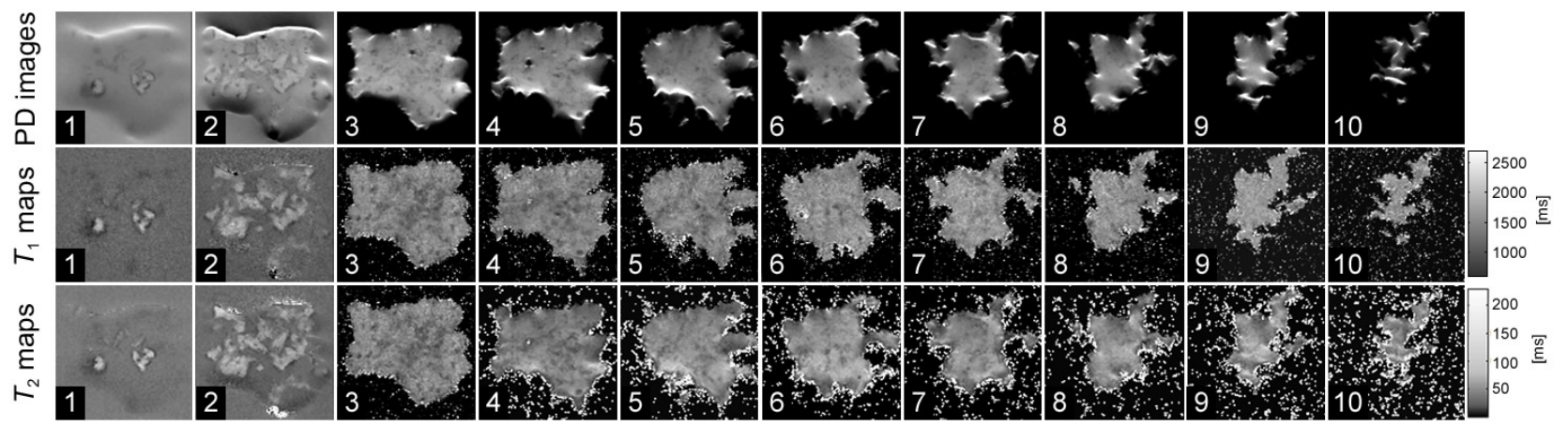

Fig.1. The ten PD-weighted images and $T_{1}, T_{2}$ maps of the coronal slices of the Norway spruce (sample No. 1).

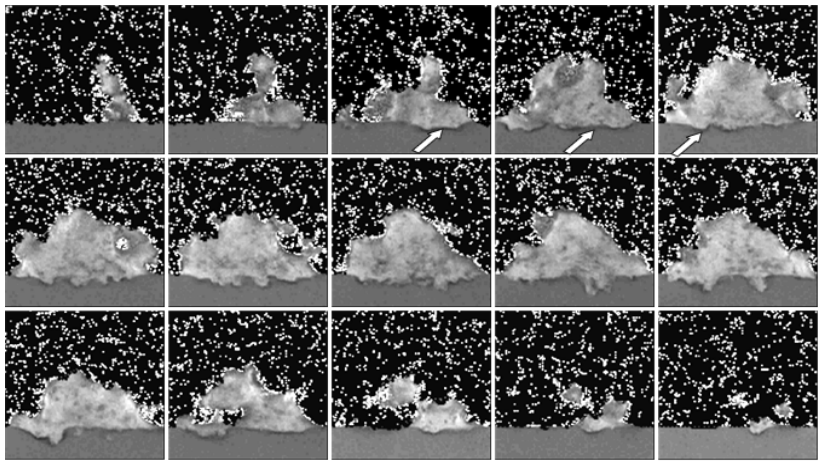

Fig.2. The $T_{2}$ maps of the axial slices of the ESEs of the Norway spruce (sample No. 1). The transition layer is indicated by the arrows.

In the acquired axial slices of the embryogenic tissue, the thin layer between the embryogenic tissue and substrate is more visible. Fig.2. shows the $T_{2}$ maps exhibiting good contrast between the transition layer, substrate, and embryogenic tissue; the layer is also clearly perceptible in the $T_{1}$ maps.

Unlike the PD images, the $T_{1}$ and $T_{2}$ maps enable us to recognize individual portions of the embryogenic tissue. Manual selection of the region containing the specific parts (the ESEs, mucilage, and transition layer) is not always reproducible; therefore, we performed automatic segmentation of the sought parts to ensure the reproducibility of the experiment. The measured data of the isolated ESEs and mucilage were used in the formation of the model to be applied for the automatic segmentation of the embryogenic tissue.

The distribution of the relaxation times $T_{1}$ and $T_{2}$ in the isolated ESEs and mucilage is shown in Fig.3. Two regions with different $T_{1}$ and $T_{2}$ can be subjectively distinguished; the relaxation time values were obtained from two slices where the isolated ESEs and mucilage are plainly visible. The regions of the ESEs and mucilage are represented by 568 and 455 manually selected pixels, respectively.

In the first step, we estimated the basic statistical values from the selected set of relaxation values. For both the ESEs and the mucilage, the sample mean values and sample standard deviations were estimated. Then, the image data (relaxation times) were approximated by means of the twocomponent GMM, and this model was also used to estimate the mean values and standard deviations. The result of approximating the three-dimensional (3D) histograms via the GMM is presented in Fig.3.; the 3D histogram shows two areas with normal distribution.

The calculated mean values of the relaxation times of the ESEs and mucilage determined for two different approaches are shown in Table 1. In the former case, we defined the statistical parameters, namely, the sample mean value and standard deviation, from randomly selected pixels inside the ESEs and mucilage; as regards the latter approach, estimations of the mean values and standard deviations were 
established from the two-component GMM applied to the same data. The mean values and standard deviations of the relaxation times $T_{1}$ and $T_{2}$ for both approaches correspond to each other; this fact confirms the assumed normal distribution of the relaxation times inside the homogeneous regions, and it also clearly points to the possibility of modeling the image data through the use of a GMM.

The second processing stage consists of the computation of the probability images, where each pixel represents the probability that a pixel belongs to the ESEs or mucilage. In the case of the two-component model, two probability images are obtained. The results acquired from one slice of sample 2 (culture) are shown in Fig.4.; here, the left image shows the pixels pertaining to the ESEs, while the right one indicates those related to the mucilage.

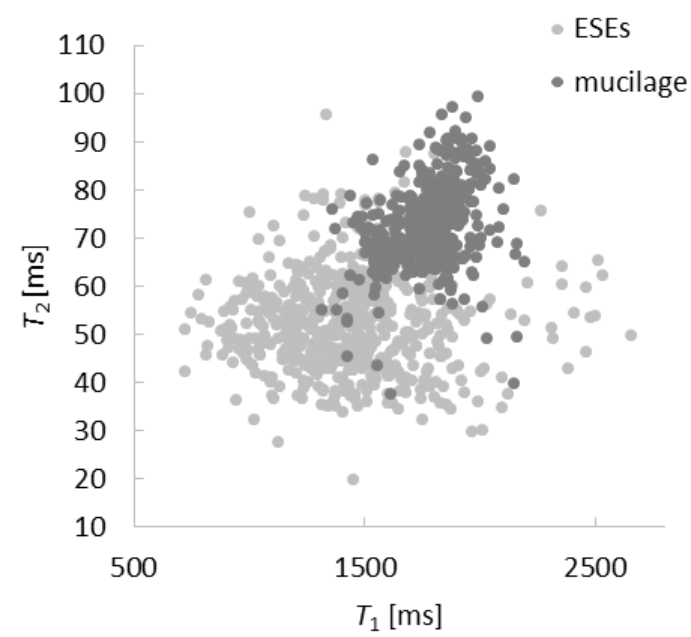

In the last processing stage, the probability images are segmented by means of the threshold method. The segmentation threshold was determined according to the $3 \sigma$ (3-sigma) principle, which is a very strict criterion; for this reason, we preferred $1 \sigma$. In the case of normal distribution, $1 \sigma$ expresses the probability of $\mathrm{p}=68.2 \%$ that a pixel really belongs to the ESEs or mucilage. The result of the thresholding is utilized for the segmentation of the original $T_{1}$ - and $T_{2}$-weighted images. The segmented regions for both samples are presented in Fig.5., where the red line defines the entire area of the analyzed embryogenic tissue, the yellow one separates the regions with the ESEs, and the blue one marks the mucilage regions; for the latter two groups, the probability of $\mathrm{p}=68.2 \%$ applies.

Fig.3. The distribution of the relaxation times $T_{1}$ and $T_{2}$ in the ESEs and mucilage regions (left); the fitted probability density function (a two-component Gaussian mixture model) of the modeled relaxation times (right)..

Table 1. The estimation of the mean values and standard deviations of the relaxation times from randomly selected pixels and from values determined by distribution function modeling via the two-component GMM.

\begin{tabular}{|c|c|c|c|c|}
\hline \multirow{2}{*}{} & \multicolumn{2}{|c|}{ Isolated mucilage } & \multicolumn{2}{c|}{ Isolated ESEs } \\
\cline { 2 - 5 } & $T_{1}[\mathrm{~ms}]$ & $T_{2}[\mathrm{~ms}]$ & $T_{1}[\mathrm{~ms}]$ & $T_{2}[\mathrm{~ms}]$ \\
\hline $\begin{array}{c}\text { Sample } \\
\text { selection }\end{array}$ & $1763 \pm 169$ & $73 \pm 8$ & $1435 \pm 318$ & $52 \pm 10$ \\
\hline GMM & $1784 \pm 124$ & $74 \pm 8$ & $1469 \pm 324$ & $53 \pm 10$ \\
\hline
\end{tabular}
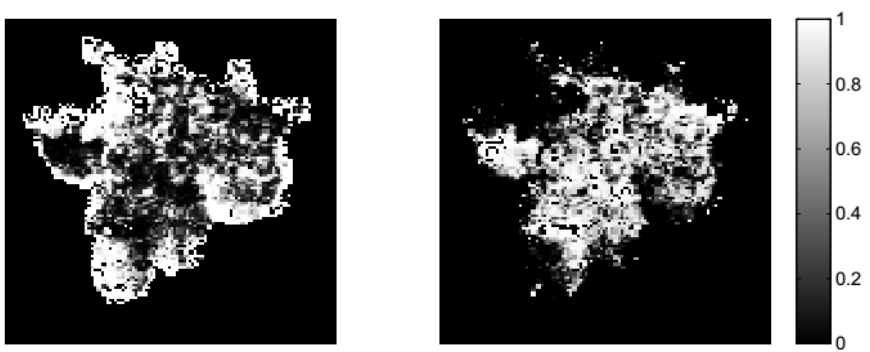

Fig.4. The probability maps of the ESEs (left) and mucilage (right).
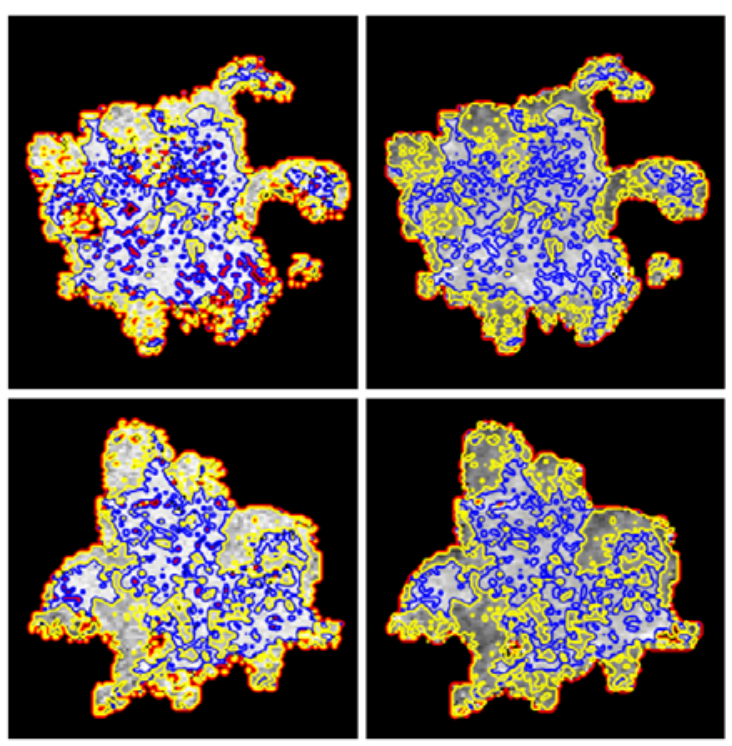

Fig.5. The segmented $T_{1}$ and $T_{2}$ maps of cultures No. 1 and No. 2 . The $T_{1}$ maps are shown on the left (top and bottom), while the $T_{2}$ ones are presented on the right (top and bottom). 
In the case of the axial slices, we expanded our twocomponent GMM to a three-component one in such a manner that the manually selected pixels in the area of the transition layer from the chosen axial slice were added to the original two-component GMM. As the separation of the transition layer is practically unfeasible, the pixels representing the transition layer in the axial slice were selected manually. The three-component GMM secures the possibility of recognizing, besides the ESEs and mucilage, also the transition layer between the embryogenic tissue and applied substrate. The distribution of the relaxation times for the ESEs, mucilage, and transition layer is shown in Fig.6. (left). The resulting approximation of the three-dimensional (3D) histograms via the three-component GMM can be seen in Fig.6. (right).

The calculated mean values of the relaxation times of the ESEs, mucilage, and transition layer are shown in Table 2. The three-component GMM was successfully applied in the axial slices, where the transition layer is visible. The probability distribution maps for the ESEs, mucilage, and transition layer can be seen in Fig.7. The final segmentation was performed with the same threshold $(p=68.2 \%)$ as in the previous case. The green contour in Fig.8. represents the region of the segmented transition layer.

The relaxometry measurements of the isolated ESEs and mucilage show that these parts of the embryogenic tissue can be well characterized using a classifier created from the relaxation times $T_{1}$ and $T_{2}$. The relaxation times of the isolated ESEs, mucilage, and the transition layer are indicated in Table 2. The elliptical scattering of the relaxation times (Fig.3.) is caused by the fact that we cannot ensure good separation of these embryogenic tissue parts, and this is especially true of the manually separated pixels of the transition layer (Fig.6.). However, we also need to note that the same MRI sequences were used for both the measurement of the discussed isolated tissue parts and the in situ measurement.

Table 2. The estimation of the mean values and standard deviations of the relaxation time values determined via distribution function modeling with the three-component GMM.

\begin{tabular}{|c|c|c|c|c|c|c|}
\hline \multirow{2}{*}{} & \multicolumn{2}{|c|}{$\begin{array}{c}\text { Isolated } \\
\text { mucilage }\end{array}$} & \multicolumn{2}{c|}{ Isolated ESEs } & \multicolumn{2}{c|}{ Transition layer } \\
\cline { 2 - 7 } & $T_{1}[\mathrm{~ms}]$ & $T_{2}[\mathrm{~ms}]$ & $T_{1}[\mathrm{~ms}]$ & $T_{2}[\mathrm{~ms}]$ & $T_{1}[\mathrm{~ms}]$ & $T_{2}[\mathrm{~ms}]$ \\
\hline \multirow{2}{*}{$\mathrm{GMM}$} & $1784 \pm$ & $74 \pm$ & $1469 \pm$ & $53 \pm$ & $929 \pm$ & $32 \pm$ \\
& 124 & 8 & 324 & 10 & 164 & 4.7 \\
\hline
\end{tabular}

After the MRI-based measuring steps, the morphology of the embryogenic tissue was displayed (Fig.9.a), b)) using optical microscopy (an Olympus SZX10 stereo microscope). The sample orientation of the embryogenic tissue is the same as in the MRI measurement (Fig.9.c)). Fig.9. contains a PD-weighted image and the images obtained via the optical microscopy technique. While the former two images show the surfaces of embryogenic tissues No. 1 and No. 2, the latter one exhibits a slice (defined volume) through the inside of embryogenic tissue No. 1 . The air bubbles in the culture are clearly visible (the white bold arrows in the PD image). In the PD-weighted image, the high-intensity regions are artefacts due to the different magnetic susceptibilities between the air and the embryogenic tissue; these artefacts do not affect the resulting $T_{1}$ and $T_{2}$ maps.
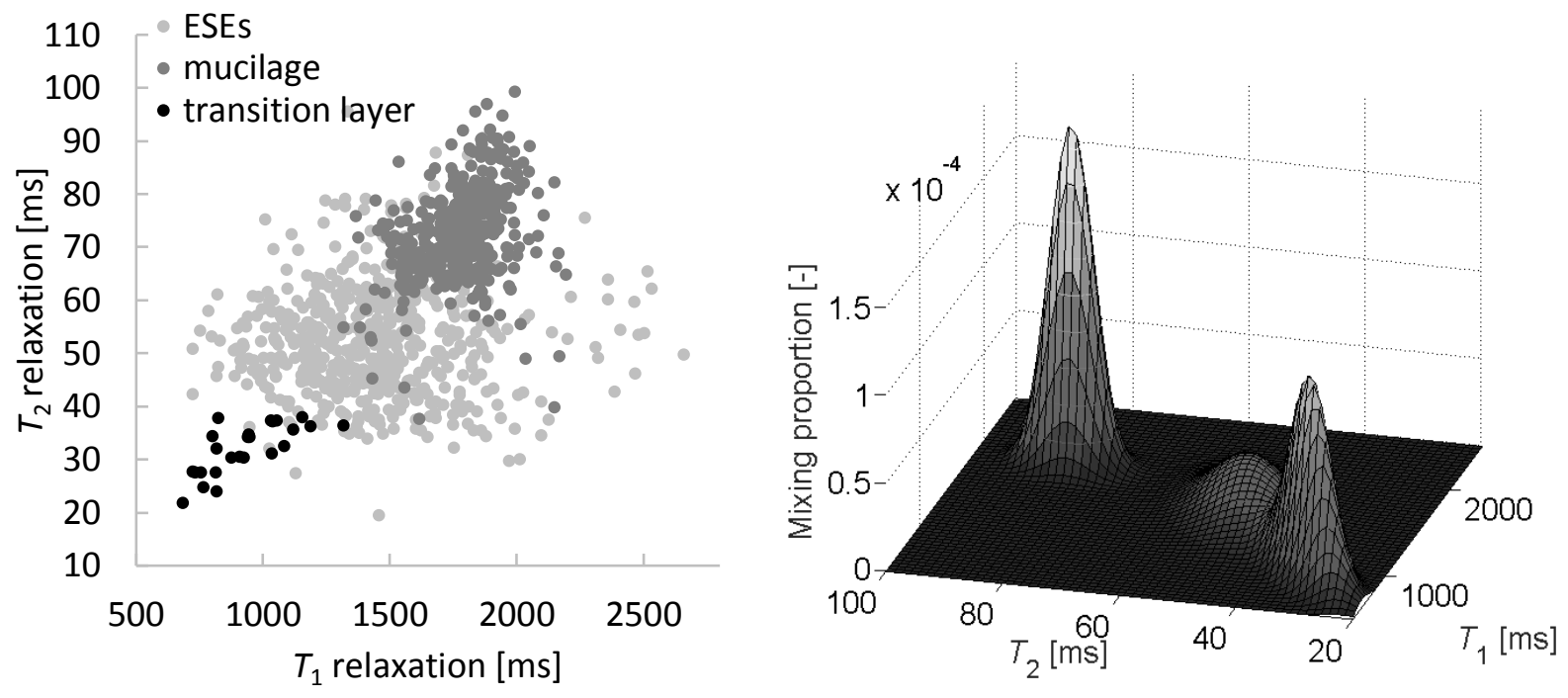

Fig.6. The distribution of the $T_{1}$ and $T_{2}$ relaxation times in the regions of the ESEs, mucilage, and transition layer (left); the fitted probability density function (a three-component Gaussian mixture model) of the modeled relaxation times (right). 

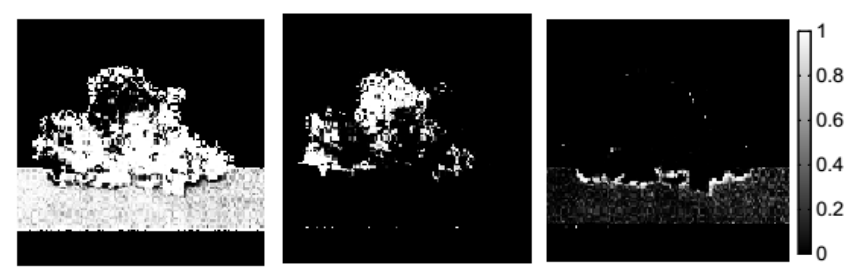

Fig.7. The probability maps of the ESEs (left), mucilage (center), and transition layer (right).
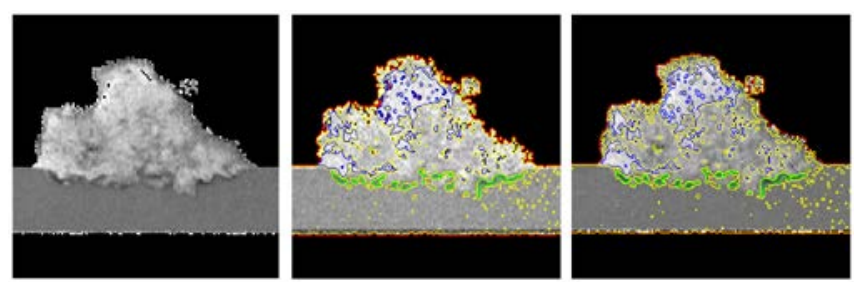

Fig.8. The original $T_{1}$ map of the axial slice (left); the segmentation results in the $T_{1}$ (centre) and $T_{2}$ (right) maps. The green line indicates the transition layer.
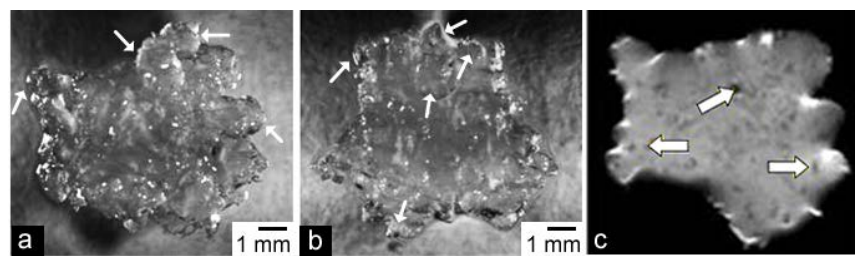

Fig.9. The microscopic images of samples No. 1 (a) and No. 2 (b); the thin white arrows show clusters of the ESEs. The PD-weighted MR image (c) of the ESEs of the Norway spruce (sample No. 1); the bold white arrows indicate the air bubbles.

\section{CONCLUSION AND DISCUSSION}

The embryogenic tissue of a conifer is characterized by the presence of bipolar structures composed of an embryonic part with densely packed embryogenic cells and a suspensor formed by long vacuolated cells [12]. A structural subcellular marker, such as the extracellular matrix surface network (ECMSN), was found to be a characteristic feature for the embryogenic tissue [26], [27]. From the botanical point of view, the ECMSN is plant mucilage. The cell wall of plants consists of dynamic cellular compartments with structural, protective, and growth regulating functions. Plant cell walls, together with cytoskeleton, determine the polarity and control the morphogenesis [28]. The external cell wall is particularly interesting due to its exposure to environmental factors; in this context, let us note that the different cellular responses mediate the adaptation of the plant to changed environmental conditions. One of such responses may lie in the formation of the ECM surface layer or network on the outer cell wall. The secretions are probably one of the factors playing a role in the cell integration and recognition process. The morphogenesis is controlled by particular morphogenic programs within which cell-to-cell communication is critical for the cellular decision-making process. Dostál [29] indicates that growth correlations become more complex and difficult during plant development. It appears likely that, in the course of the embryogenesis, the ECM constitutes an important structural integrity agent.

The role of the ECMSN during morphogenesis remains unclear; however, the cell adhesion, protective function, and coordinating developmental stage have all been explained.[30]. The analysis of the chemical composition of the ECMSN performed with different solvents and enzymes revealed differences between the species and identified the relevant proteins. Other researchers used specific antibodies to identify the AGPs and pectin epitopes in the ECMSN.[31], [30]. Generally, AGPs are plant cell surface macromolecules belonging to the subfamily of hydroxyprolin-rich glycoproteins; they can act as signaling macromolecules and are involved in the vegetative, reproductive, and cellular growth and development [19]. Verdeil et al. [30] indicate that the chemical composition and structural arrangement of the ECMSN on a cell surface play a significant role in morphogenic processes.

The measurement of embryogenic cultures via magnetic resonance imaging is beneficial for embryogenic tissue characterization. In more general terms, the intensity of a PD-weighted image in a voxel is proportional to the amount of ${ }^{1} \mathrm{H}$ nuclei (a quantitative summary of the number of protons per unit tissue) in the measured tissue. The water holding capacity of the AGPs of the ECM may reflect other physiological roles. The AGPs' ability to hydrate may be also important in their capacity to prevent injuries and to resist drying out [17]. For many years, the ECM was dismissed as a relatively inert "ground substance", whose sole function was packing; however, it has now become clear that the ECM is significantly more complex than previously presumed and that the interaction with the surrounding matrix is one of the major elements to control cell behavior. [32].

The results obtained through MRI relaxometry show the possibility of distinguishing between the ESEs, ECMSN (mucilage), and transition layer in an embryogenic tissue; for these recognition purposes, uniform classifiers are applied. The classifiers consist only of the $T_{1}$ and $T_{2}$ relaxation times. The dispersion of the $T_{2}$ relaxation time for the ESEs, mucilage, and transition layer is low compared to the $T_{1}$ indicator; thus, the $T_{2}$ maps are more advantageous for the actual embryogenic tissue differentiation. The distribution of the ESEs, mucilage, and transition layer was obtained using automatic segmentation. The mucilage (ECMSN) is then distributed unevenly in the embryogenic tissue. Regions with a minimum mucilage volume also appear in the space between the cells (the ESEs). In the case of MR imaging applied to represent an embryogenic tissue, the quantification of the amount of ESEs and mucilage within a voxel is unfeasible.

Relaxometry measurements in combination with the subsequent automatic multi-parametric segmentation (based on the $T_{1}$ and $T_{2}$ relaxation times) provide non-destructive differentiation between the cells and mucilage in an embryogenic tissue. In the case of contiguous slices, three- 
dimensional distribution of the cells and mucilage can be visualized. The function of the transition layer remains unclear; thus, the aim of further investigation is to perform a detailed analysis of the chemical composition and properties of the transition layer between the embryogenic callus and the medium. In a narrower sense, within the given context, we seek to define the effect of the transition layer on the growth and development of the culture. Changes of the relaxation times $T_{1}$ and $T_{2}$ during cultivation may help us to characterize the chemical and physical changes in the ESEs. Moreover, the times $T_{1}$ and $T_{2}$ may serve as an indicator of the effect of stress on the developmental somatic embryos in an embryogenic culture in situ. Relaxometry measurements appear to constitute an interesting and useful methodological element in the research into the structure of embryogenic tissues.

\section{ACKNOWLEDGMENT}

The research was supported by grants of the Czech Science Foundation (GA14-22777S, GA13-09086S) and by the European Commission and the Ministry of Education, Youth, and Sports (projects No. CZ.1.05/2.1.00/01.0017, LO1212).

\section{REFERENCES}

[1] Mac Fall, J.S., Van As, H. (1996). Magnetic resonance imaging of plants. In Nuclear Magnetic Resonance in Plant Biology. The American Society of Plant Physiologists, 33-76.

[2] Scheenen, T., Vergeldt, F., Heemskerk, A., Van As, H. (2007). Intact plant magnetic resonance imaging to study dynamics in long-distance sap flow and flowconducting surface area. Plant Physiology, 144, 11571165.

[3] Ionenko, I., Anisimov, A., Dautova, N. (2010). Effect of temperature on water transport through aquaporins. Biologia Plantarum, 54, 488-494.

[4] Pu, Y., Chen, F., Ziebell, A., Davison, B., Ragauskas, A. (2009). NMR characterization of C3H and HCT down-regulated alfalfa lignin. BioEnergy Research, 2, 198-208.

[5] Zulak, K., Weljie, A., Vogel, H., Facchini, P. (2008). Quantitative 1H NMR metabolomics reveals extensive metabolic reprogramming of primary and secondary metabolism in elicitor-treated opium poppy cell cultures. BMC Plant Biology, 8, 5.

[6] Lambert, J., Lampen, P., von Bohlen, A., Hergenroder, R. (2006). Two- and three- dimensional mapping of the iron distribution in the apoplastic fluid of plant leaf tissue by means of magnetic resonance imaging. Analytical and Bioanalytical Chemistry, 384, 231-236.

[7] Glidewell, S., Möller, M., Duncan, G., Mill, R., Masson, D., Williamson, B. (2002). NMR imaging as a tool for noninvasive taxonomy: Comparison of female cones of two Podocarpaceae. New Phytologist, 154, 197-207.
[8] Šupálková, V., Petřek, J., Baloun, J., Adam, V., Bartušek, K., Trnková, L., Beklová, M., Diopan, V., Havel, L., Kizek, R. (2007). Multi-instrumental investigation of affecting of early somatic embryos of spruce by cadmium (II) and lead (II) ions. Sensors, 7, 743-759.

[9] Šebánek, J., Sladký, Z., Procházka, S. (1991). Experimental Morphogenesis and Integration of Plants: Terofal. 1st Edition. Prague, Czech Republic: Academia; Elsevier.

[10] Dostál, R. (1967). On Integration in Plants. 1st Edition. Harvard University Press.

[11] Hřib, J., Vooková, B., Neděla, V. (2015). Imaging of native early embryogenic tissue of Scots pine (Pinus sylvestris L.) by ESEM. Open Life Sciences, 10, 285290.

[12] Šamaj, J., Salaj, T., Matúšová, R., Salaj, J., Takáč, T., Šamajová, O., Volkmann, D. (2008). Arabinogalactanprotein epitope Gal4 is differentially regulated and localized in cell lines of hybrid fir (Abies alba x Abies cephalonica) with different embryogenic and regeneration potential. Plant Cell Reports, 27, 221229.

[13] Neděla, V., Hřib, J., Vooková, B. (2012). Imaging of early conifer embryogenic tissues with the environmental scanning electron microscope. Biologia Plantarum, 56, 595-598.

[14] Neděla, V., Hřib, J., Havel, L., Runštuk, J. (2013) Early state of spruce somatic embryos in native state observed using the ESEM and Cryo-SEM. Microscopy and Microanalysis, 19 (suppl. 2), 20-21.

[15] Neděla, V., Tihlaříková, E., Hřib, J. (2015). The lowtemperature method for study of coniferous tissues in the environmental scanning electron microscope. Microscopy Research Techniques, 78 (1), 13-21.

[16] Egertsdotter, U., von Arnold, S. (1995). Importance of arabinogalactan proteins for the development of somatic embryos of Norway spruce (Picea abies). Physiologia Plantarum, 93, 334-345.

[17] Clarke, A., Anderson, R.L., Stone, B. (1979). Form and function of arabinogalactans and arabinogalactanproteins. Phytochemistry, 18, 521-540.

[18] Karácsonyi, Š., Pätoprstý, V., Kubačková, M. (1998). Structural study on arabinogalactan-proteins from Picea abies L. Karst. Carbohydrate Research, 307, 271-279.

[19] Seifert, G., Roberts, K. (2007). The biology of arabinogalactan proteins. Annual Review of Plant Biology, 58, 137-161.

[20] Mikulka, J., Hutova, E., Korinek, R., Marcon, P., Dokoupil, Z., Gescheidtova, E., Havel, L., Bartusek, K. (2016). MRI-based visualization of the relaxation times of early somatic embryos. Measurement Science Review, 16, 54-61.

[21] von Arnold, S. (1987). Improved efficiency of somatic embryogenesis in mature embryos of Picea abies (L.) Karst. Journal of Plant Physiology, 128, 233-244. 
[22] Havel, L., Durzan, D. (1996). Apoptosis during diploid parthenogenesis and early somatic embryogenesis of Norway spruce. International Journal of Plant Sciences, 157, 8-16.

[23] Vlašínová, H., Mikulecký, M., Havel, L. (2003). The mitotic activity of Norway spruce polyembryonic culture oscillates during the synodic lunar cycle. Biologia Plantarum, 47, 475-476.

[24] Bloch, F. (1946). Nuclear Induction. Physical Review, 70, 460-473.

[25] Xiong, T., Zhang, L., Yi, Z. (2016). Double Gaussian mixture model for image segmentation with spatial relationship. Journal of Visual Communication and Image Representation, 34, 135-145.

[26] Dubois, T., Dubois, J., Guedira, M., Diop, A., Vasseur, J. (1992). SEM characterization of an extracellular matrix around somatic proembryos in roots of Cichorium. Annals of Botany, 70, 119-124.

[27] Šamaj, J., Bobák, M., Blehová, A., Krištin, J, Auxtová-Šamajová, O. (1995). Developmental SEM observations of an extracellular matrix in embryogenic calli of Drosera rotundifolia and Zea mays. Protoplasma, 186, 45-49.
[28] Baluška, F., Šamaj, J., Wojtaszek, P., Volkmann, D., Menzel, D. (2003). Cytoskeleton-plasma membranecell wall continuum in plants. Emerging links revisited. Plant Physiology, 133, 482-491.

[29] Dostál, R. (1959). O celistvosti rostliny (On Integration in Plants). Prague, Czech Republic: SZN. (in Czech)

[30] Verdeil, J., Hocher, V., Huet, C., Grosdemange, F., Escoute, J., Ferrière, N., Nicole, M. (2001). Ultrastructural changes in coconut calli associated with the acquisition of embryogenic competence. Annals of Botany, 88, 9-18.

[31] Šamaj, J., Baluška, F., Bobák, M., Volkmann, D. (1999). Extracellular matrix surface network of embryogenic units of friable maize callus contains arabinogalactan-proteins recognized by monoclonal antibody JIM 4. Plant Cell Reports, 18, 369-374.

[32] Davies, J. (2001). Extracellular matrix. In Encyclopedia of Life Sciences. Nature Publishing Group.

Received August 23, 2016. Accepted January 25, 2017. 